\title{
The effect of Bu Zhong Yi Qi decoction on simulated weightlessness-induced muscle atrophy and its mechanisms
}

\author{
MU ZHU ${ }^{1,2}$, ZHONGYANG LIU $^{3}$, MINGZE GAO $^{1}$, YAN ZHANG ${ }^{1}$, YUHENG LI $^{2}$, SHUKUAN LING ${ }^{2}$, \\ PENGFEI ZHANG ${ }^{2}$, CHENYANG ZHAO ${ }^{2}$, LIJUN JIANG ${ }^{2}$, YU LIU $^{2}$, \\ QI LI ${ }^{2}$, DONG LI ${ }^{3}$, SUMIN HU$^{1}$ and YINGXIAN LI ${ }^{2}$ \\ ${ }^{1}$ Department of Chinese Materia Medica, School of Preclinical Medicine, Beijing University of \\ Chinese Medicine, Beijing 100019; ${ }^{2}$ State Key Laboratory of Space Medicine Fundamentals and Applications, \\ China Astronaut Research and Training Center, Beijing 100094; ${ }^{3}$ State Key Laboratory of Proteomics, \\ Beijing Proteome Research Center, Institute of Radiation Medicine, Beijing 102206, P.R. China
}

Received August 10, 2016; Accepted May 11, 2017

DOI: $10.3892 / \mathrm{mmr} .2017 .7287$

\begin{abstract}
Microgravity has been previously demonstrated to induce skeletal muscle atrophy, loss of muscle force and disorders in myogenesis and metabolism. Current pharmacological strategies exhibit poor efficacy. Bu Zhong Yi Qi decoction (BZ) is a well-known traditional Chinese medicine decoction used for myasthenia gravis. In the present study, its effect on unloading induced muscle atrophy was investigated. The mousetail suspension model was used to simulate weightlessness induced muscle atrophy. The results indicated that BZ could significantly protect muscles from simulated weightlessness-induced atrophy. To elucidate the underlying mechanisms, drugCIPHER-CS methods were introduced to predict its potential targets, significantly enriched pathways and biological processes. The results demonstrated that the calcium signaling pathway, citrate cycle, biosynthetic and lipid metabolic process are affected by BZ. Among the targets, nuclear receptor corepressor 1 (NCoR1) is one of the most important proteins involved in myogenesis and metabolism. The results indicated that BZ significantly downregulated NCoR 1 expression, and further induced muscle differentiation and metabolism by regulating NCoR1-associated gene expression in vivo and in vitro. In summary, the present study
\end{abstract}

Correspondence to: Professor Yingxian Li, State Key Laboratory of Space Medicine Fundamentals and Applications, China Astronaut Research and Training Center, 26 Beiqing Road, Beijing 100094, P.R. China

E-mail: yingxianli@aliyun.com

Professor Sumin Hu, Department of Chinese Materia Medica, School of Preclinical Medicine, Beijing University of Chinese Medicine, 11 3rd Northeast Ring Road, Beijing 100019, P.R. China E-mail: husm@bucm.edu.cn

Key words: traditional Chinese medicine, protective effects, simulated weightlessness-induced muscle atrophy, network pharmacology indicated that may be effective in combating weightlessness-induced muscle atrophy. Combined with bioinformatics, the underlying mechanism for this decoction was investigated, which provided an improved understanding of this decoction.

\section{Introduction}

Prolonged space flight can cause rapid and severe atrophy in skeletal muscle due to the weightlessness experienced. It has been demonstrated that real and simulated spaceflight results in a loss of muscle mass and reductions in muscle strength and endurance in humans and rodents (1-4). Satellite cells are skeletal muscular stem cells that are the primary source for muscle regeneration. Satellite cell dysfunction, representing impaired proliferation capacity or increased apoptosis, is suggested to contribute to the decreasing muscle regeneration in atrophy $(5,6)$. Oxidative stress can be one of the triggers of muscle atrophy (7), while alterations of mitochondrial biogenesis and dynamics have been observed in prolonged unloading of muscle $(8,9)$. Countermeasures against these causes may be a possible resolution for weightlessness-induced muscle atrophy. Effective prevention of weightlessness-induced muscle atrophy required for space missions of increasing durations. Chinese medicine has been used previously in countermeasures for muscle-associated diseases. In traditional Chinese medicine (TCM), it is believed that the spleen governs the muscle, and is closely associated with qi (10). In TCM it is thought that in space, as the earth qi is reduced, the spleen is relatively weak. Bu Zhong Yi Qi decoction (BZ) predominantly consists of Radix Ginseng, Radix Angelicae and has been reported to improve spleen function and is used in treatment of myasthenia gravis (11). In the present study, the effects of BZ on weightlessness-induced muscle atrophy and its mechanisms were investigated. BZ is additionally commonly used in clinical practice for chronic fatigue and chronic atrophic gastritis, and is widely produced (12). Large scale epidemiological studies in clinical trials have been conducted, suggesting that BZ has a clear effect on improving muscle function (11). In addition, investigations have been conducted into the bioactivities of monomers present in BZ. 
Hexadecanoic acid from $\mathrm{BZ}$ was reported to induce bone marrow mesenchymal stem cell proliferation depending on the retinoic acid receptor pathway and upregulated the mRNA levels of insulin-like growth factor-1 (13). However, individual pharmacological effects of the monomers present do not fully account for the effects of this formula in general, and the pharmacology of BZ remains unclear. Bioinformatic methods provide predictions of drug-target interactions, identifying underlying molecular mechanisms. The drugCIPHER-CS method, which was developed by Zhao and Li (14), offers an effective method to understand the complicated multitargeted mechanisms of TCM herbal formulas. The two basic principles of this method are that drugs with similar chemical structures are likely to bind functional-associated proteins, and functional associations between the proteins can be measured by their distance in the protein interaction network (15). It has been demonstrated as a reliable method to obtain potential targets for decoctions (16). In addition, it is possible to analyze the significantly enriched pathways using Kyoto Encyclopedia of Genes and Genomes (KEGG) biological pathway analysis and Gene Ontology (GO) analysis to identify the biological processes involved in these targets. In the present study, it was identified that BZ was able to protect from simulated weightlessness-induced loss of muscle mass, with a reduction in the gastrocnemius (GAS) cross sectional area and a decrease in twitch force. The results of the bioinformatics analysis identified the main biological pathways and processes involved the effect of BZ. Experimental verification is required in order to further reveal the underlying mechanisms.

\section{Materials and methods}

Animal studies. Male Kunming (KM) mice ( $\mathrm{n}=24$; age, 8 weeks old; weight, 25 g; Beijing Vital River Laboratory Animal Technology Co, Ltd., Beijing, China) were divided into control (Ctrl), hindlimb-suspended (HS) and BZ-fed-hindlimb-suspended (BZ) groups ( $\mathrm{n}=8 \mathrm{mice} / \mathrm{group}$ ). All mice were maintained in a temperature $\left(23^{\circ} \mathrm{C}\right)$ and humidity (50-70\%)-controlled specific pathogen free (SPF) animal facility with a $12 \mathrm{~h}$ light/dark cycle and were given free access to food and water. HS and BZ mice were suspended by attaching a harness to the proximal end of the tail. The hindlimb suspension model was adopted to simulate unloading-induced muscle atrophy, as previously described (17). The mice were suspended at a $30^{\circ}$ angle to the floor to prevent contact with any supportive surfaces, with only the forelimbs maintained in contact with the grid floor, which allowed the mice to move and access food and water freely. HS and BZ mice were suspended for 14 days. BZ mice were fed with $\mathrm{BZ}$ during this period and 7 days previously, while HS and Ctrl mice were fed with water. No necrosis of hindlimb or unexpected death of animals was observed during the experimental procedure. At the end of suspension, mice were euthanized for muscle isolation by intraperitoneal injection with $1.5 \%$ pentobarbital sodium (Sigma-Aldrich; Merck Millipore, Darmstadt, Germany). The experimental procedures were approved by the Animal Care and Use Committee of China Astronaut Research and Training Center (Beijing, China), and all animal studies were performed according to the approved guidelines for the care and use of live animals.
Drug preparation and dose consideration. According to its origin, Treatise on the Spleen and Stomach (18), the BZ consists of eight Chinese herbs, at a ratio of 5:5:3:5:2:4:2:3, as follows: Radix Astragalus membranaeus (Huangqi); Radix Panax ginseng (Renshen); Radix Angelica Sinensis (Danggui); Glycyrrhiza uralensis (Gancao); Cimicifuga foetida (Shengma); Herba Bupleurumchinense (Chaihu); Citrus sinensis Osbeck (Chenpi); and Atractylodes macrocephala (Baizhu), respectively. Herbs were purchased from Tongren Tang Pharmacy (Beijing, China). These 8 types of herbs, at a total weight of $89 \mathrm{~g}$, were extracted twice in $890 \mathrm{ml}$ boiling water for $1 \mathrm{~h}$. A liquor solution was formed after extraction. The liquid was then concentrated to obtain a paste, which was collected and water was added to a total volume of $100 \mathrm{ml}$, so that the liquid contained $0.89 \mathrm{~g}$ herbal medicine $/ \mathrm{ml}$. A brown medical liquor was finally obtained with $\mathrm{pH}$ of 7.4. Based on the recommended human daily dosage of $89 \mathrm{~g} /$ day, according to the equivalent conversion between humans and mice by body weight, a dosage of $5.93 \mathrm{mg} / \mathrm{g}$ was used in the current study.

Drug-containing serum preparation. BZ was fed to male Wistar rats for 5 days ( $\mathrm{n}=5$; weight, 293-301 g; supplied by Beijing Vital River Laboratory Animal Technology Co., Ltd.). Rats were maintained in a temperature $\left(23^{\circ} \mathrm{C}\right)$ and humidity (50-70\%)-controlled SPF animal facility with a $12 \mathrm{~h}$ light/dark cycle and were given free access to food and water. The dosage of BZ used was $11.87 \mathrm{mg} / \mathrm{g}$, which was twice the dosage for the mice during tail-suspension period as previously described. Serum was taken on the fifth day $2 \mathrm{~h}$ after the rats were fed with BZ.

Ex vivo assessment of skeletal muscle contractile properties. Following 14 days of hindlimb suspension, the GAS was isolated from distal tendons intact and nylon sutures were attached to the tendons. The other end of the string was attached to the MedLabBio-Signal Processing System (MedLab-U/4C501H; Nanjing Medease Science and Technology Co., Ltd, Nanjing, China; http:/www.medease .com.cn). Muscles were adjusted perpendicularly to the operation table to obtain the maximal twitch force and bathed with PBS solution to remain wet. Ex vivo muscle stimulation and data analysis was performed using the Medlab system. Peak isometric twitch force was analyzed from isometric contraction, and isometric twitch contractions were stimulated in muscles at a frequency of every $50 \mathrm{~Hz}$, with the stimulation current varying from 0 to $10 \mathrm{~V}$.

Histological analysis. Cryosections of GAS were fixed in $4 \%$ paraformaldehyde in PBS for $10 \mathrm{~min}$ at $4^{\circ} \mathrm{C}$ and washed in PBS. After fixing of the samples with $15 \%$ goat serum (Sigma-Aldrich; Merck $\mathrm{KGaA}$ ) at room temperature, the sections were incubated overnight at $4^{\circ} \mathrm{C}$ with mouse monoclonal anti-laminin primary antibodies (CWP223;1:50; Beijing Kangwei Century Biotechnology Co., Ltd., Beijing, China). The primary antibody was detected by incubation at room temperature for $1 \mathrm{~h}$ with fluorescein-conjugated affinipure goat anti-mouse immunoglobulin G (TA130003; 1:200; OriGene Technologies, Inc., Rockville, MD, USA.) diluted in BSA. Three independent GAS cryosections and 8 fibers per section were counted for cross-sectional area (CSA) analysis. 
Table I. Primers used for reverse transcription-quantitative polymerase chain reaction analysis.

\begin{tabular}{lll}
\hline Gene name & \multicolumn{1}{c}{ Forward primer } & \multicolumn{1}{c}{ Reverse primer } \\
\hline NCoR1 & 5'-CTGGTCTTTCAGCCACCATT-3' & 5'-CCTTCATTGGATCCTCCATC-3' \\
MEF2C & 5'-TCCATCAGCCATTTCAACAA-3' & 5'-GTTACAGAGCCGAGGTGGAG-3' \\
MEF2D & 5'-CTTCGCGTAACCGAGGATT-3' & 5'-GGGACTAGTGATCAGTTCATGG-3' \\
Myoglobin & 5'-CTGACGAAGGCCACTTTGCACCTCTG-3' & 5'-GCACAAGATCCCGGTCAAGTACCTGGAG-3' \\
MCK & 5'-TGAACTCGCCCGTCAGGCTGTTGAG-3' & 5'-GATGTCATCCAGACTGGGGTGGACAACC-3' \\
Pax7 & 5'-CGATTAGCCGAGTGCTCAGA-3' & 5'-GGAGGTCGGGTTCTGATTCC-3' \\
Myogenin & 5'-GGTGCCCAGTGAATGCAACT-3' & 5'-GCTGTCCACGATGGACGTAA-3' \\
MyHC & 5'-CCAAGGGCCTGAATGAGGAG-3' & 5'-GCAAAGGCTCCAGGTCTGAG-3' \\
Citrate synthase & 5'-GGAGCCAAGAACTCATCCTG-3' & 5'-TCTGGCCTGCTCCTTAGGTA-3' \\
LCAD & 5'-GTAGCTTATGAATGTGTGCAACTC-3' & 5'-GTCTTGCGATCAGCTCTTTCATTA-3' \\
VEGF-B & 5'-AGCCACCAGAAGAAAGTGGT-3' & 5'-GCTGGGCACTAGTTGTTTGA-3' \\
VEGF-A 121 & 5'-AACGATGAAGCCCTGGAGTG-3' & 5'-TGAGAGGTCTGGTTCCCGA-3' \\
GAPDH & 5'-TCACCACCATGGAGAAGGC-3' & 5'-GCTAAGCAGTTGGTGGTGCA-3' \\
COX2 & 5'-CATCCCCTTCCTGCGAAGTT-3' & 5'-TTTAAGTCCACTCCATGGCCC-3' \\
UCP2 & 5'-TGGCAGGTAGCACCACAGG-3' & 5'-CATCTGGTCTTGCAGCAACTCT-3'
\end{tabular}

NCoR1, nuclear receptor corepressor 1; MEF2C, myocyte-specific enhancer factor 2C; MEF2D, myocyte-specific enhancer factor 2D; MCK, muscle creatine kinase; Pax7, paired Box 7; MyHC, myosin heavy chain; LCAD, long chain acyl-CoA dehydrogenase; VEGF-B, vascular endothelial growth factor B; VEGF-A 121, vascular endothelial growth factor A; GAPDH, glyceraldehyde-3-phosphate dehydrogenase; $\mathrm{COX} 2$, cytochrome c oxidase subunit 2; UCP2, uncoupling protein 2.

Reverse transcription-quantitative polymerase chain reaction $(R T-q P C R)$. Total RNA was extracted from GAS muscles using TRIzol reagent (Invitrogen; Thermo Fisher Scientific, Inc., Waltham, MA, USA) following the manufacturer's instructions. A total of $3 \mu \mathrm{g}$ RNA was reverse-transcribed using the PrimeScript RT reagent kit (Perfect Real Time) with gDNA Eraser (Takara Bio, Inc., Otsu, Japan). Gene expression was assessed using qPCR with SYBR Premix Ex Taq II (TliRNaseH Plus; Takara Bio., Inc.) according to the manufacturer's protocol. qPCR values were normalized with U6 and the primers used are listed in Table I. The following thermal settings were used: $95^{\circ} \mathrm{C}$ for $30 \mathrm{sec}$ followed by 40 cycles of $95^{\circ} \mathrm{C}$ for $5 \mathrm{sec}$ and $60^{\circ} \mathrm{C}$ for $30 \mathrm{sec}$. Relative expression values were calculated using the comparative threshold cycle $(\Delta \Delta \mathrm{Cq})$ method in accordance with previous guidelines $(19,20)$.

Cell culture. C2C12 myoblasts were obtained from ATCC (Manassas, VA, USA) and were cultured in Dulbecco's modified Eagle's medium (DMEM) supplemented with $10 \%$ fetal bovine serum (FBS) and antibiotics $(100 \mathrm{U} / \mathrm{ml}$ penicillin and $100 \mu \mathrm{g} / \mathrm{ml}$ streptomycin). The cells were differentiated in DMEM containing $2 \%$ horse serum [M\&C Gene Technology (Beijing) Ltd., Beijing, China]. To compare the effect of serum from BZ-fed rats, we treated the cells with $2 \%$ serum from normal rat as a $\mathrm{Ctrl}$.

Cell proliferation assay. C2C12 myoblasts were seeded into 96-well plates with growth medium for $12 \mathrm{~h}$, then were cultured with differentiation medium (DMEM containing 2\% horse serum) for 3 days. Cell proliferation was detected subsequent to incubation with $10 \%$ MTS (BioVision, Inc., Milpitas,
CA, USA) for 5-6 h under $485 \mathrm{~nm}$ UV light. The experiments were repeated three times.

Drug-target prediction and analyses. Firstly, the composite compounds of BZ were identified from the literature. The drugCIPHER-CS method was applied to predict the potential drug-targets of the composite compounds. drugCIPHER-CS was developed by Zhao and $\mathrm{Li}$ (14), and can infer drug-targets in the genome-wide scale and achieves good prediction performance. Given a set of known drug (drug space)-target (target space) interactions, for a query drug and a candidate target-gene, drugCIPHER-CS will measure the likelihood of their interaction based on the correlation between the query drug's structure similarity with the drug space and the candidate gene's functional similarity with the target space. For each query compound, drugCIPHER-CS ranks its potential targets according to the order of the decreasing interaction possibility (14). In the current study, known drug-target interactions were obtained from the DrugBank database (version: May, 2011) (21), the chemical structure similarity is calculated based on compounds' MOLPRINT 2D descriptors and Tanimoto coefficient. The human protein interaction network was constructed by integrating the protein interaction data from: Human Protein Reference Database (HPRD; release 9.0, http://www.hprd.org/) (22), the Biological General Repository for Interaction Datasets (BioGRID; version 3.0.66, https://thebiogrid.org/) (23), the IntAct Molecular Interaction Database (IntAct; version 20100628; http://www .ebi.ac.uk/intact/) (24), the Molecular INTeraction database (MINT; version 20100505; http://mint.bio.uniroma2 it/) (25), the Database of Interacting Proteins (DIP; version 20100614; http://dip.doe-mbi.ucla.edu) (26) and the Protein 
Data Bank (PDB; http://www.rcsb.org/pdb/home/home.do) provided by Gibson and Goldberg (27).

After obtaining the potential targets of chemical components of BZ, hypergeometric cumulative distribution test is used to investigate the significantly enriched biological pathways and GO biological processes among these potential targets. A biological pathway or GO biological process was considered to be significantly enriched with drug-targets of BZ when the corresponding upper-tailed P-value of hypergeometric cumulative distribution test was $<0.05$. In the present study, biological pathway data are from KEGG database (http://www.genome.jp/kegg/; last updated Oct 16, 2012) (28) and $\mathrm{GO}$ biological process annotation of human proteins downloaded from GO project website (version Sep 9, 2011; http://www.geneontology.org/) (29). The network topological analysis of targets is based on the integrated human protein interaction network as mentioned above.

Statistical analysis. Data are presented as the mean \pm standard deviation. Statistical significance was determined by the two-tailed t-test using GraphPad Prism 5 (GraphPad Software, Inc., La Jolla, CA, USA). $\mathrm{P}<0.05$ was considered to indicate a statistically significant difference.

\section{Results}

Mice fed with $B Z$ are resistant to simulated microgravity-induced muscle atrophy. To examine the role of BZ in skeletal muscle under HS, BZ was administrated to hindlimb-suspended mice for 14 days. Thereafter, the ratios of soleus (SOL) and GAS muscle weight to the whole-body weight were analyzed in the Ctrl, HS and BZ group mice. Hindlimb suspended mice exhibited over 30 and 12\% reductions in relative SOL and GAS muscle mass, respectively, in comparison with Ctrl mice. However, hindlimb-suspended mice fed with BZdemonstrated 20 and 10\% increase in comparison with HS mice (Fig. 1A). This suggested that BZ partly protected hindlimb suspended mice from skeletal muscle atrophy. Immunostaining for laminin of GAS cross sectional area (CSA) delineated a decrease of GAS fiber size in the HS mice in comparison to Ctrl. In contrast, BZ muscle fiber size increased in comparison with HS (Fig. 1B). Morphometric analysis of GAS CSA highlighted a significant difference in myofiber size between HS and BZ muscles, confirming that BZ mice were resistant to muscle atrophy (Fig. 1B). GAS muscles were dissected and attached for assessment of isometric contractile properties. Representative twitch force traces of the muscles were presented (Fig. 1C). Isometric twitch force was significantly increased in the muscles from BZ fed mice compared with their hindlimb suspended counterparts (Fig. 1C).

Target prediction and analyses of the composite compounds of BZ. To elucidate the pharmacological mechanism of BZ, the potential drug-targets of the composite compounds of BZ were predicted and analyzed by bioinformatics analysis. First, by a comprehensive review of the literature, 238 non-redundant composite compounds were identified for 8 herbs of BZ. Subsequently, drugCIPHER-CS method was used to predict the potential targets of these compounds. For a query compound,
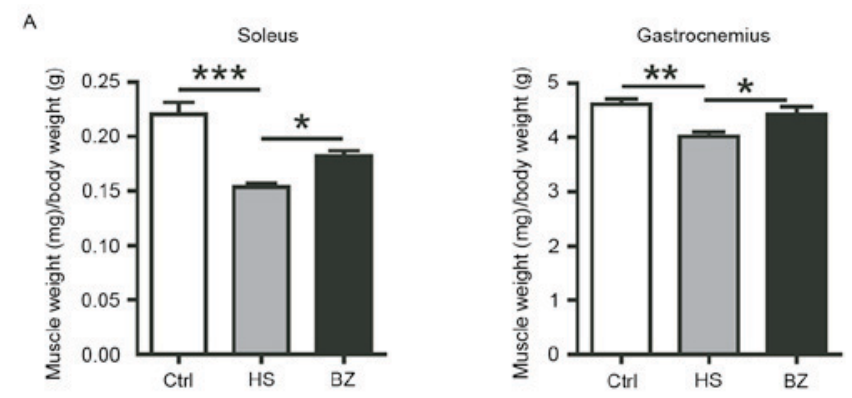

B
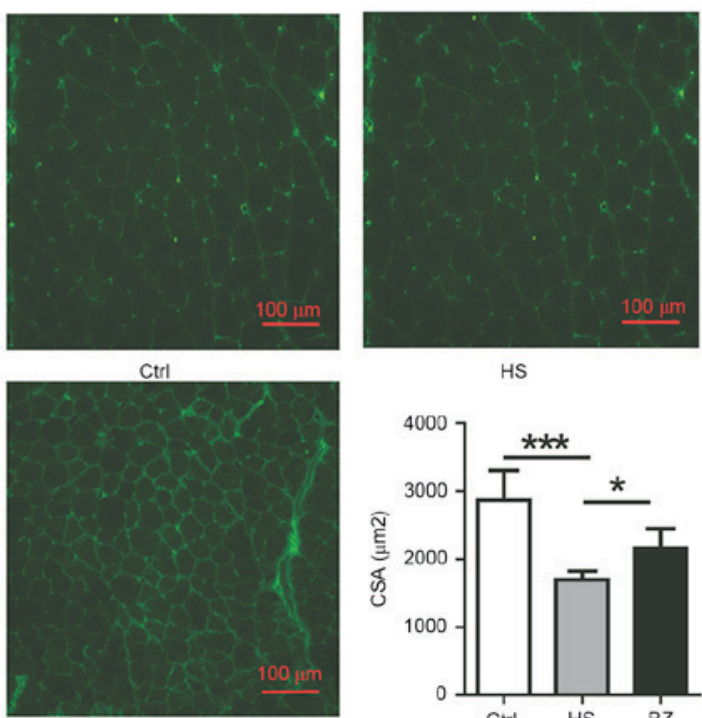

HS

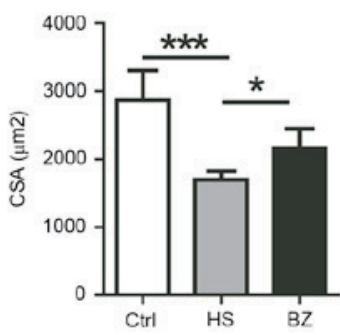

$\mathrm{Bz}$
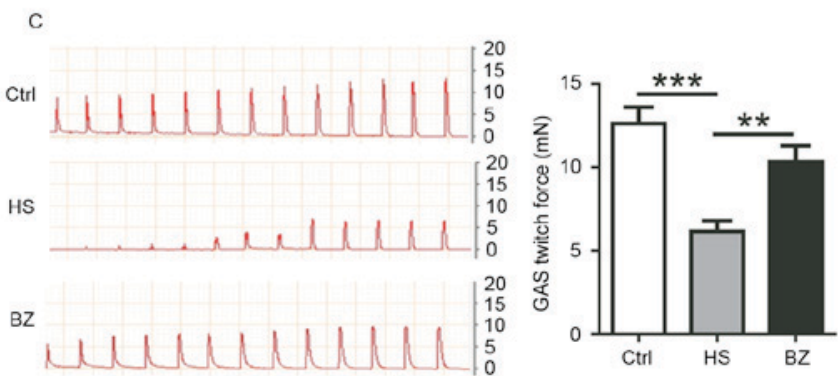

Figure 1. The effect of BZ on skeletal muscle atrophy induced by tail suspension in mice. KM mice in HS and BZ group were suspended for 14 days, during which time BZ group mice were treated with BZ. (A) The ratios of SOL and GAS muscle weight to body weight in the mice of $\mathrm{Ctrl}$, tail suspended and BZ treatment group. Data are presented as mean \pm standard deviation. ${ }^{*} \mathrm{P}<0.05$; ${ }^{* *} \mathrm{P}<0.01 ;{ }^{* * *} \mathrm{P}<0.005 ; \mathrm{n}=8$ for each group. (B) Immunostaining for laminin of GAS muscle in Ctrl, tail suspension and BZ fed mice. Data are presented as the mean \pm standard deviation. ${ }^{*} \mathrm{P}<0.05 ;{ }^{* *} \mathrm{P}<0.01 ;{ }^{* * *} \mathrm{P}<0.005$. A total of 3 independent GAS cryosections and 8 fibers per section were counted for CSA analysis, $\mathrm{n}=8$ for each group. (C) Ex vivo contractile properties of Ctrl, tail suspension and BZ fed mice, twitch force of GAS muscle from mice. Data are presented as the mean \pm standard deviation. ${ }^{*} \mathrm{P}<0.05 ;{ }^{* *} \mathrm{P}<0.01 ;{ }^{* * *} \mathrm{P}<0.005$; $\mathrm{n}=8$ for each group. BZ, Bu Zhong Yi Qi decoction; KM, Kunming; HS, hindlimb suspended; SOL, soleus; GAS, gastrocnemius; Ctrl, control.

a score for each candidate target was given by measuring the compound-target interaction possibility. When the top $0.1 \%$ candidate targets were considered, 451 potential targets of BZ were obtained. Candidate genes were on average targeted by 5.8 compositive compounds.

Furthermore, bioinformatics analysis was conducted for these candidate targets. It was identified that there were 17 significantly enriched KEGG biological pathways among 
Table II. Significantly enriched KEGG biological pathways among top $0.1 \%$ candidate target genes of BZ composite compounds.

\begin{tabular}{llr}
\hline KEGG pathway number and name & P-value & Coverage \\
\hline hsa04020-Calcium signaling pathway & $5.06 \times 10^{-5}$ & 0.095505618 \\
hsa00020-Citrate cycle & $4.67 \times 10^{-4}$ & 0.1875 \\
hsa04260-Cardiac muscle contraction & $9.95 \times 10^{-4}$ & 0.1125 \\
hsa00590-Arachidonic acid metabolism & $2.42 \times 10^{-3}$ & 0.120689655 \\
hsa00510-N-Glycan biosynthesis & $3.32 \times 10^{-3}$ & 0.130434783 \\
hsa00640-Propanoate metabolism & $3.77 \times 10^{-3}$ & 0.151515152 \\
hsa04080-Neuroactive ligand-receptor interaction & $8.15 \times 10^{-3}$ & 0.059602649 \\
hsa00561-Glycerolipid metabolism & $1.42 \times 10^{-2}$ & 0.111111111 \\
hsa00512-O-Glycan biosynthesis & $1.49 \times 10^{-2}$ & 0.133333333 \\
hsa00830-Retinol metabolism & $1.65 \times 10^{-2}$ & 0.09375 \\
hsa05010-Alzheimer's disease & $2.01 \times 10^{-2}$ & 0.065088757 \\
hsa04115-p53 signaling pathway & $2.32 \times 10^{-2}$ & 0.086956522 \\
hsa05410-Hypertrophic cardiomyopathy & $2.42 \times 10^{-2}$ & 0.078651685 \\
hsa05414-Dilated cardiomyopathy & $2.84 \times 10^{-2}$ & 0.076086957 \\
hsa00190-Oxidative phosphorylation & $2.97 \times 10^{-2}$ & 0.066666667 \\
hsa05412-Arrhythmogenic right ventricular cardiomyopathy & $3.52 \times 10^{-2}$ & 0.078947368 \\
hsa00970-Aminoacyl-tRNA biosynthesis & $4.20 \times 10^{-2}$ & 0.097560976 \\
\hline
\end{tabular}

Pathways whose corresponding upper-tailed P-value of the hypergeometric cumulative distribution was $<0.05$ were deemed to be significantly enriched by candidate target genes. Those pathways were ranked according to the order of the increasing P-values. The coverage for each pathway was referred to as the fraction of candidate target genes among all the pathway member genes. KEGG, Kyoto Encyclopedia of Genes and Genomes; BZ, Bu Zhong Yi Qi decoction.

these 451 candidate targets, including calcium signaling pathway and tricarboxylic acid cycle (TCA cycle; Table II). The calcium signaling pathway is well established in muscle dystrophy, which is activated by ROS to produce $\mathrm{Ca}^{2+}$ entry, resulting in a significant effect of muscle damage (30). TCA cycle flux and adenosine triphosphate (ATP) synthesis rate, TCA cycle and oxidative phosphorylation pathway genes were downregulated in muscle atrophy (31-34).

In addition, GO biological process enrichment analysis was conducted and this indicated that candidate targets were significantly enriched in biological processes including biosynthetic process, cellular nitrogen compound metabolic process, lipid metabolic process, cellular amino acid metabolic process and response to stress (Table III). Protein and amino acid metabolism is well known to be disrupted during muscle atrophy (35). In addition, fatty acid metabolism and lipid oxidation has been identified to be impaired in the majority of muscle disorders (36-38). The significantly enriched biological pathways and processes are potential target pathways and proteins of BZ, providing important evidence for elucidating the molecular mechanism of BZ.

To further determine the key candidate targets of BZ, the topological property of these candidate targets was assessed in the protein interaction network. The degree of a protein was referred to as its number of interaction partners in the protein interaction network, which could reflect the biological importance of a protein. The higher the degree of a protein was, the higher its biological importance. The degree was used to rank the candidate targets, proteins with relatively high degrees included splicing factor 3a subunit 1 (SF3A1), COP9 signalosome subunit 5 (COPS5) and other targets (Table IV). SF3A1, necessary for the in vitro conversion of $15 \mathrm{~S}$ U2 snRNP into an active $17 \mathrm{~S}$ particle that performs pre-mRNA splicing (39), has been demonstrated to have stability in relative quantity in skeletal muscle (40). Genome wide association studies indicated that COPS5 interacted with key regulators of beef cattle carcass intermuscular fat (41). These proteins may serve key roles in muscle physiology and BZ-induced protection. Among the targets predicted, NCoR1 was identified to have a high degree and betweeness centrality in the protein interaction network (Table IV), and is a well-known gene closely associated with muscle growth and oxidative function.

BZ reduces NCoR 1 expression levels and promotes myogenesis under simulated weightlessness-induced muscle atrophy. To establish whether NCoR1 is a target for BZ to counteract muscle atrophy, the transcription levels of $\mathrm{NCoR} 1$ were detected in hindlimb-suspended and BZ-fed mice in GAS by RT-qPCR analysis. As presented in the results, NCoR1 expression was increased in the muscles of HS mice, and was markedly downregulated upon BZ administration (Fig. 2A), confirming that BZ may inhibit the upregulation of NCoR1 expression in muscles under conditions of simulated microgravity.

As reported previously, the expression of Mef2c and Mef2d negatively correlated with NCoR1 expression. These are the key transcription factors involved in the expression of muscle remodeling genes (42-44). The mRNA levels were reduced in HS mice compared with that of the Ctrl mice. Following BZ treatment, the expression levels of Mef2c were markedly increased. In line with this, mRNA levels of two MEF2 targets, 
Table III. Significantly enriched GO biological process term among top $0.1 \%$ candidate target genes of BZ composite compounds.

\begin{tabular}{|c|c|c|}
\hline GO term ID & GO term name & P-value \\
\hline GO:0009058 & Biosynthetic process & $2.27 \times 10^{-9}$ \\
\hline GO:0034641 & $\begin{array}{l}\text { Cellular nitrogen compound } \\
\text { metabolic process }\end{array}$ & $5.20 \times 10^{-6}$ \\
\hline GO:0044281 & $\begin{array}{l}\text { Small molecule metabolic } \\
\text { process }\end{array}$ & $2.22 \times 10^{-5}$ \\
\hline GO:0006629 & Lipid metabolic process & $5.33 \times 10^{-5}$ \\
\hline GO:0019748 & Secondary metabolic process & $1.45 \times 10^{-4}$ \\
\hline GO:0003013 & Circulatory system process & $2.89 \times 10^{-3}$ \\
\hline GO:0051276 & Chromosome organization & $1.08 \times 10^{-2}$ \\
\hline GO:0006520 & $\begin{array}{l}\text { Cellular amino acid metabolic } \\
\text { process }\end{array}$ & $1.14 \times 10^{-2}$ \\
\hline GO:0051186 & Cofactor metabolic process & $1.46 \times 10^{-2}$ \\
\hline GO:0006399 & tRNA metabolic process & $2.28 \times 10^{-2}$ \\
\hline GO:0006950 & Response to stress & $4.69 \times 10^{-2}$ \\
\hline GO:0042592 & Homeostatic process & $4.87 \times 10^{-2}$ \\
\hline \multicolumn{3}{|c|}{$\begin{array}{l}\text { The top } 12 \text { candidate target genes are significantly enriched with } \\
\text { genes annotated with a GO term when its corresponding upper-tailed } \\
\text { P-value of hypergeometric cumulative distribution was }<0.05 \text {. These } \\
\text { GO terms are ranked according to the order of the increasing P-values. } \\
\text { GO, gene ontology; BZ, Bu Zhong Yi Qi decoction. }\end{array}$} \\
\hline
\end{tabular}

$\mathrm{Mb}$ and Mck, which were two markers of myogenesis, were upregulated in GAS subsequent to administration with BZ (Fig. 2B). These results further confirmed that could stimulate myogenesis, and NCoR1 mediated the effect of BZ on muscle atrophy.

Muscle differentiation has been previously reported to be impaired during hindlimb suspension-induced muscle atrophy (45). Pax 7, myogenin and myosin heavy chain (MyHC) are markers of muscle proliferation and differentiation. The expression levels of Pax 7 and myogenin were reduced in HS mice. By contrast, BZ-fed mice displayed a marked increase in the mRNA expression of Pax 7, myogenin and MyHC. Pax 7 and myogenin expression increased 3-fold and MyHC increased by $>44$ fold compared with that of HS mice (Fig. 2C), indicating that BZ-fed mice were resistant to muscle atrophy by enhancing muscle differentiation and growth.

$B Z$ reduces NCoR1 levels and promotes myogenesis and oxidative function in vitro. To further confirm the regulation of BZ on myogenesis and its mechanism, the effect of serum from BZ-fed rats on myoblasts was investigated. Subsequent to incubation of the $\mathrm{C} 2 \mathrm{C} 12$ myoblasts in medium with serum containing BZ, the expression of NCoR1 was significantly reduced in comparison with the cells incubated with the serum from rats that did not receive BZ (Fig. 3A). Subsequently, the effect of $\mathrm{BZ}$ on the proliferation and differentiation of $\mathrm{C} 2 \mathrm{C} 12$ cells in vitro was investigated. Following treatment ofC $2 \mathrm{C} 12$ cells with the medium containing serum from the rats fed with $\mathrm{BZ}$, the cell numbers were markedly higher than those without
Table IV. Drug target prediction and analysis.

\begin{tabular}{lcc}
\hline Potential & $\begin{array}{c}\text { Degree in } \\
\text { the protein } \\
\text { interaction } \\
\text { network }\end{array}$ & $\begin{array}{c}\text { Interaction partners } \\
\text { in the protein } \\
\text { interaction network } \\
\text { (gene ID: gene symbol) }\end{array}$ \\
\hline 10291:SF3A1 & 126 & 9939:RBM8A \\
10987:COPS5 & 113 & 6095:RORA \\
7520:XRCC5 & 111 & 6418:SET \\
9611:NCoR1 & 95 & $7704:$ ZTB16 \\
2091:FBL & 85 & $3190:$ HNRNPK \\
6601:SMARCC2 & 82 & 10262:SF3B4 \\
408:ARRB1 & 79 & 1855:DVL1 \\
6633:SNRPD2 & 79 & 10250:SRRM1 \\
6605:SMARCE1 & 76 & $7030:$ TFE3 \\
6602:SMARCD1 & 74 & 1024:CDK8 \\
86:ACTL6A & 73 & 5469:MED1 \\
7421:VDR & 72 & 8204:NRIP1 \\
50813:COPS7A & 70 & 22919:MAPRE1 \\
5705:PSMC5 & 70 & 2099:ESR1 \\
\hline
\end{tabular}

The proteins with the highest degree/betweeness centrality are referred to as those with the top 5\% degree/betweeness centrality in the protein interaction network. SF3A1, splicing factor 3a subunit 1; COPS5, COP9 signalosome subunit 5; XRCC5, X-ray repair cross complementing 5; NCoR1, nuclear receptor corepressor 1; FBL, fibrillarin; SMARCC2, SWI/SNF related, matrix associated, actin dependent regulator of chromatin subfamily c member 2; ARRB1, arrestin $\beta 1$; SNRPD2, small nuclear ribonucleoprotein D2 polypeptide; SMARCE1, SWI/SNF related, matrix associated, actin dependent regulator of chromatin, subfamily e, member 1; SMARCD1, SWI/SNF related, matrix associated, actin dependent regulator of chromatin, subfamily d, member 1; ACTL6A, actin-like 6A; VDR, vitamin D receptor; COPS7A, COP9 signalosome subunit 7A; PSMC5, proteasome 26S subunit, ATPase 5.

BZ treatment (Fig. 3B). The expression levels of muscle differentiation marker genes were also upregulated following incubation in BZ serum (Fig. 3C). These results demonstrated that $\mathrm{BZ}$ promoted the proliferation of $\mathrm{C} 2 \mathrm{C} 12$ in addition to enhancing the expression of muscle differentiation marker genes such as myogenin and MyHC.

$\mathrm{BZ}$ treatment was indicated to enhance muscle peak force of BZ-fed mice (Fig. 1C), which is consistent with the results of bioinformatic analysis (Tables II and III). The enriched potential KEGG biological pathways included calcium signaling pathway, TCA cycle, cardiac muscle contraction and arachidonic acid metabolism. TCA cycle is the energy generating reaction through the oxidation of carbohydrates, fats and proteins in the form of ATP. Calcium levels in the mitochondrial matrix are necessary for the activation of isocitrate dehydrogenase, one of the key regulatory enzymes of the TCA cycle (46). The significantly enriched GO biological processes, which indicated the functional distribution of those candidate targets, included several metabolic processes, including cellular nitrogen compound, small molecule and lipid and secondary metabolic process. These results indicated that the mechanism of BZ may be closely associated with the energy 
A

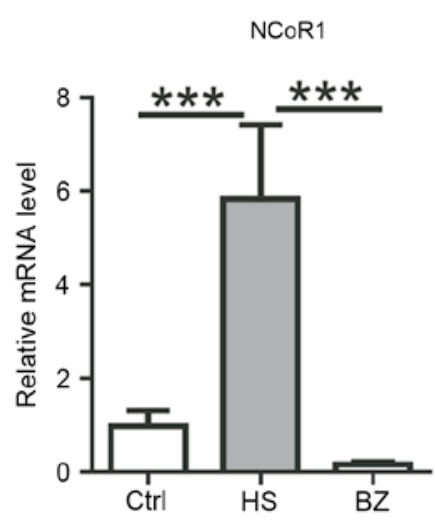

B

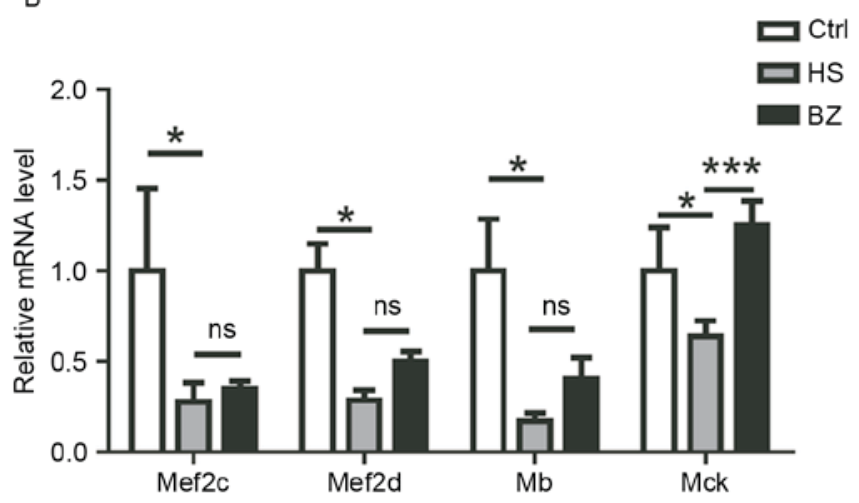

C
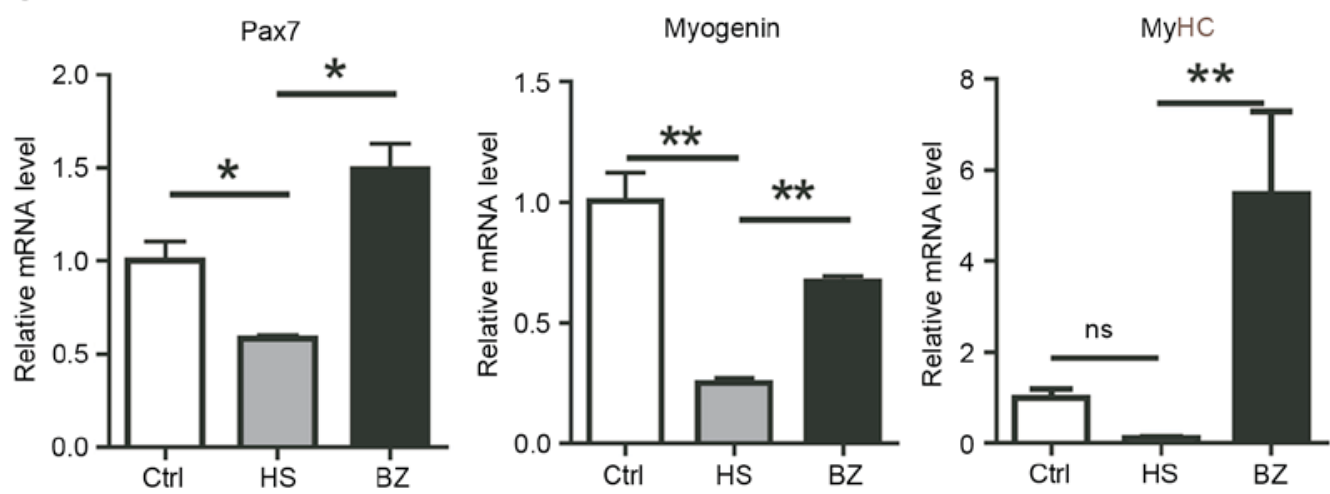

Figure 2. BZ reduces NCoR1 expression levels and promotes myogenesis in mice. (A) The expression of NCoR1 in GAS in the mice of Ctrl, tail suspended and BZ fed mice was detected by RT-qPCR. The values are normalized to Ctrl. (B) The expression of target genes of NCoR1 in GAS in Ctrl, tail suspended and BZ fed mice were detected by RT-qPCR. The values are normalized to the Ctrl. (C) The expression of Pax7, Myogenin and MyHC of GAS in Ctrl, tail suspended and BZ fed mice, detected by RT-qPCR. The values are normalized to the Ctrl. Data are presented as the mean \pm standard deviation. ${ }^{*} \mathrm{P}<0.05$; ${ }^{* *} \mathrm{P}<0.01 ; \mathrm{n}=8$ for each group. BZ, Bu Zhong Yi Qi decoction; NCoR1, nuclear receptor corepressor 1; GAS, gastrocnemius; Ctrl, control; RT-qPCR, reverse transcription-quantitative polymerase chain reaction; HS, hindlimb suspended; ns, non-significant.

metabolism. The expression levels of genes associated with the oxidative capacity of muscle cells in mitochondria were measured. The mitochondrial DNA marker, cyclooxygenase 2, normalized by genomic DNA marker, uncoupling protein 2 , was significantly higher in $\mathrm{BZ}$-incubated $\mathrm{C} 2 \mathrm{C} 12$, indicative of increased mitochondrial content (Fig. 3D). NCoR1 targeting genes citrate synthase, long-chain acyl-CoA dehydrogenase, vascular endothelial growth factor isoform a-121 and -b were analyzed by RT-qPCR (Fig. 3E). These genes are involved in the regulation of mitochondrial function. Their mRNA expression levels in cells incubated with BZ containing rat serum were significantly increased in comparison with that of rat $\mathrm{Ctrl}$ serum-incubated cells. These results suggest that the aerobic capacity of myocytes was promoted by downregulation of NCoR1 following BZ treatment.

\section{Discussion}

In the present study, it was demonstrated that BZ could protect from simulated weightlessness-induced muscle atrophy. The muscle mass and isometric twitch force was observed to be ameliorated. Using the drugCIPHER-CS method, the potential targets of the compounds in BZ were predicted, and the interaction between these targets and potential pathways were analyzed. The results indicated that calcium signaling pathways, TCA cycle and lipid metabolism process serve an important role in the network. NCoR1 is one of the important targets that is involved in muscle differentiation and metabolism (47). The experiments of the present study further verified their associations with muscle remodeling.

BZ was originally prescribed by Li Dongyuan in his book Treatise on the Spleen and Stomach (18). BZ is believed in TCM to 'tonify qi and replenish the spleen', leading to an improvement to muscle health (18).

In the present study, BZ was observed to regulate calcium signaling, TCA cycle, lipid metabolism process and NCoR1 protein expression by drugCIPHER-CS. Calcium signaling pathway and TCA cycle were identified to be involved in muscle metabolism and remodeling. Slow twitch oxidative muscle fibers maintain relatively high levels of $\mathrm{Ca}^{2+}$ concentrations, while fast twitch fibers require high-amplitude $\mathrm{Ca}^{2+}$ transients and lower ambient $\mathrm{Ca}^{2+}$ levels. The coupling of ATP production through TCA cycle, which skeletal muscle function is largely reliant on, to the requirement of a contracting muscle is ensured by $\mathrm{Ca}^{2+}$ on aerobic metabolism. In addition, Calcineurin has been demonstrated to be required for muscle regeneration and hypertrophy (48).

Muscle wasting caused by spaceflight or unloading, is associated with a decreased number of quiescent and proliferating 

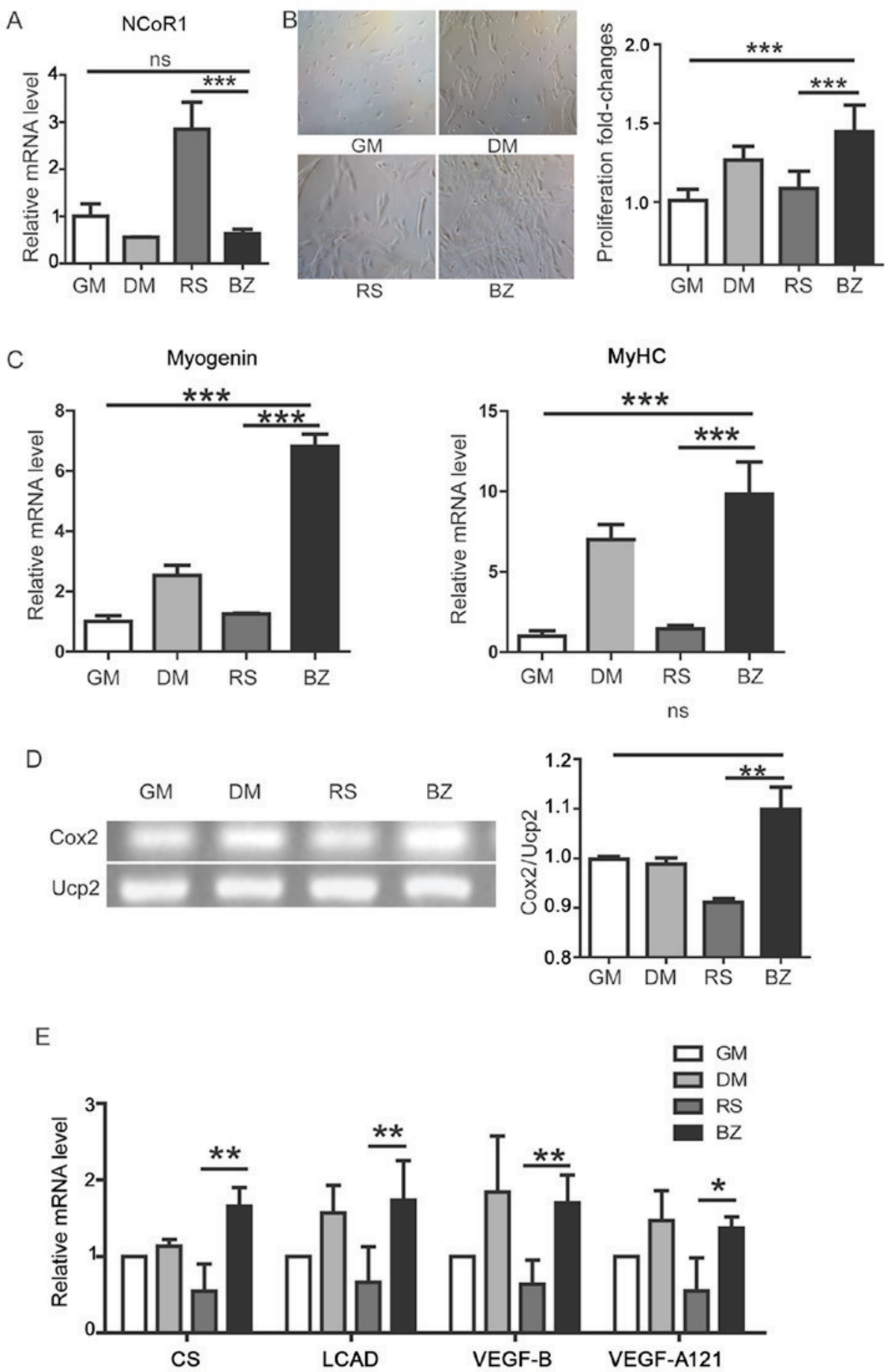

Figure 3. BZ could reduce NCoR1 level and promote myogenesis and oxidative function. (A) The expression of NCoR1 in C2C12 cells cultured in GM, DM, high glucose DMEM with 4\% RS or high glucose DMEM with 4\% rat BZ serum for 3 days, was detected by RT-qPCR. The values are normalized to Ctrl. Data are presented as the mean \pm standard deviation. " $\mathrm{P}<0.05 ;{ }^{* * * *} \mathrm{P}<0.005 ; \mathrm{n}=8$ for each sample. (B) Light microscopic images and MTS assay for the proliferation of $\mathrm{C} 2 \mathrm{C} 12$ cells cultured in GM, DM, RS and BZ for 3 days. (Cellular experiments were repeated in triplicate). (C) Expression of myogenin and MyHC in C2C12 cells cultured in GM, DM, RS and BZ for 3 days, was detected by RT-qPCR. The values are normalized to the Ctrl. Data are presented as the mean \pm standard deviation. "P<0.05; ${ }^{* * *} \mathrm{P}<0.005$; GM/DM, n=3; RS/BZ, $\mathrm{n}=4$. (D) Relative mitochondrial DNA content (Cox2) in C2C12 cells cultured in GM, DM, RS and BZ for 3 days was measured and normalized by genomic DNA content (Ucp2). $n=3$. (E) The expression of target genes of NCoR1 in different groups of $\mathrm{C} 2 \mathrm{C} 12$ cells cultured in GM, DM, RS and BZ for 3 days, were detected by RT-qPCR. The values are normalized to CtrlC2C12 cells. Data are presented as the mean \pm standard deviation. "P $<0.05 ;{ }^{* * *} \mathrm{P}<0.005 ; \mathrm{GM} / \mathrm{DM}, \mathrm{n}=3$; RS/BZ, $\mathrm{n}=4$. BZ, Bu Zhong Yi Qi decoction; NCoR1, nuclear receptor corepressor 1; GM, growth medium; DM, differentiation medium; DMEM, Dulbecco's modified Eagle's medium; RS, rat serum; RT-qPCR, reverse transcription-quantitative polymerase chain reaction; Ctrl, control; Cox2, cyclooxygenase 2; ns, non-significant.

satellite cells, which begin the repairing process by differentiating and fusing with each other. In addition, satellite cell transplantation appears to be a clinical application for multiple muscle atrophies $(49,50)$.
The impairment of oxidative metabolism and signs of mitochondrial dysfunction serves a major role in weightless muscle atrophy (51). Previous studies indicated downregulation of metabolic enzymes and decreasing mitochondrial 
oxidative capacity appeared in the later phase of atrophic muscles $(52,53)$. In addition, it has been demonstrated that the activation of mitochondrial fission is sufficient to lead to muscle wasting $(54,55)$.

It has been previously observed that NCoR 1 is a 'master modulator' of the mitochondrial metabolism in muscle, which can reprogram muscle metabolism from a glycolytic to a more oxidative mitochondrial state, in addition, muscle-specific NCoR1 knockout mice exhibit increased muscle quantity and oxidative profile (47). NCoR1 docks histone deacetylases, such as HDAC3 (56), negatively correlated with the expression of MEF2, a transcription factor controlling muscle differentiation and development (57). Chinese herbs have been identified to be closely associated with the regulation of muscle physiology. Astragali radix has been identified to exhibit protective and therapeutic effects on tail-suspension induced SOL muscle atrophy. These effects are associated with the control of increased mATPase activity (58). A previous study indicated that ginsenosideRb1 stimulated adiponectin signaling through upregulation of AdipoR1 and AdipoR2 proteins in $\mathrm{C} 2 \mathrm{C} 12$ muscle cells, in addition, glucose transporter 4, a crucial membrane protein transporting glucose into myocytes, was promoted to translocate to the cell surface (59). Adiponectin signaling has been well established in the modulation of glucose levels and stimulation of fatty acid oxidation in myocytes (60). In addition, mice fed with Angelica exhibited prolonged physical exercise duration and higher blood glucose and muscle glycogen level, indicating that Angelica (61) improved energy utilization and exercise performance in mice. Angelica polysaccharides additionally promoted skeletal muscle satellite cell proliferation and stem cell factor receptor, c-kit, expression (62). These herbs are also included in the formula of BZ.

The therapeutic application of BZ provides a novel means to counteract unloading-induced skeletal muscle atrophy. In the present study, through in vitro and in vivo experiments, it was established that BZ could protect from weightlessness-induced muscle atrophy and promote the proliferation and differentiation of myoblast cells. Its effect on muscle remodeling is by activating signaling pathways to reprogram gene expression to sustain muscle performance. This new understanding of the molecular mechanism of BZ makes the design of therapeutic compounds with improved pharmacological properties for the treatment of weightlessness-induced muscle atrophy possible.

\section{Acknowledgements}

The authors would like to thank Professor Jingping Song, from the China Astronaut Research and Training Center, for the help with cell culture. In addition, they would like to thank Mr. Guanghan Kan and Ms. Hongqing Song, from the China Astronaut Research and Training Center, for the help with animal experimental preparation. The present study was financially supported by the National Natural Scientific Foundation of China (project nos. 31170811, 31271225, 31325012, 30500663 and 11475035) and 1226 Major Project (project no. AWS16J018).

\section{References}

1. West JB: Man in space. News Physiol Sci 1: 189-192, 1986.
2. Sandoná D, Desaphy JF, Camerino GM, Bianchini E, Ciciliot S, Danieli-Betto D, Dobrowolny G, Furlan S, Germinario E, Goto K, et al: Adaptation of mouse skeletal muscle to long-term microgravity in the MDS mission. PLoS One 7: e33232, 2012.

3. Tesch PA and Berg HE: Effects of spaceflight on muscle. J Gravit Physiol 5: P19-P22, 1998.

4. Nemirovskaia TL, Shenkman BS and Krasnov IB: Effect of long-term hypergravitation on the skeletal-muscular tissue in rats. Ross Fiziol Zh Im I M Sechenova 91: 113-121, 2005 (In Russian).

5. Teixeira E and Duarte JA: Skeletal muscle loading changes its regenerative capacity. Sports Med 46: 783-792, 2016.

6. Snijders T, Nederveen JP, McKay BR, Joanisse S, Verdijk LB, van Loon LJ and Parise G: Satellite cells in human skeletal muscle plasticity. Front Physiol 6: 283, 2015.

7. Calvani R, Joseph AM, Adhihetty PJ, Miccheli A, Bossola M, Leeuwenburgh C, Bernabei R and Marzetti E: Mitochondrial pathways in sarcopenia of aging and disuse muscle atrophy. Biol Chem 394: 393-414, 2013

8. Liu J, Peng Y, Cui Z, Wu Z, Qian A, Shang P, Qu L, Li Y, Liu J and Long J: Depressed mitochondrial biogenesis and dynamic remodeling in mouse tibialis anterior and gastrocnemius induced by 4-week hindlimb unloading. IUBMB Life 64: 901-910, 2012.

9. Wagatsuma A, Kotake N, Kawachi T, Shiozuka M, Yamada S and Matsuda R: Mitochondrial adaptations in skeletal muscle to hindlimb unloading. Mol Cell Biochem 350: 1-11, 2011.

10. Huangdi N: The yellow emperor's inner cannon: Basic questions. Phoenix Press, London, 2012.

11. Liu XY, Chen SL and Zhang WX: Clinical study of strengthening pi and nourishing shen therapy combined with Western medicine on patients with glucocorticoid resistant myasthenia gravis. Zhongguo Zhong Xi Yi Jie He Za Zhi 30: 271-274, 2010 (In Chinese).

12. Zhao AB, Yu B, Wu XL, Cao KJ, Li EQ, Li QM and Chen XY: Protective effects on myelosuppression mice treated by three different classic Chinese medicine formulae. Pharmacogn Mag 7: 133-140, 2011

13. Chen DF, Li X, Xu Z, Liu X, Du SH, Li H, Zhou JH, Zeng HP and Hua ZC: Hexadecanoic acid from Buzhong Yiqi decoction induced proliferation of bone marrow mesenchymal stem cells. J Med Food 13: 967-970, 2010.

14. Zhao S and Li S: Network-based relating pharmacological and genomic spaces for drug target identification. PLoS One 5: e11764, 2010

15. Wang Y, Liu Z, Li C, Li D, Ouyang Y, Yu J, Guo S, He F and Wang W: Drug target prediction based on the herbs components: The study on the multitargets pharmacological mechanism of qishenkeli acting on the coronary heart disease. Evid Based Complement Alternat Med 2012: 698531, 2012.

16. Wang Y, Li C, Liu Z, Shi T, Wang Q, Li D, Wu Y, Han J, Guo S, Tang B and Wang W: DanQi Pill protects against heart failure through the arachidonic acid metabolism pathway by attenuating different cyclooxygenases and leukotrienes B4. BMC Complement Altern Med 14: 67, 2014.

17. Fitts RH, Metzger JM, Riley DA and Unsworth BR: Models of disuse: A comparison of hindlimb suspension and immobilization. J Appl Physiol (1985) 60: 1946-1953, 1986.

18. Li DY: The Treatise on the Spleen and Stomach. Liaoning Science \& Technology Publishing House, Shenyang, 1997.

19. Livak KJ and Schmittgen TD: Analysis of relative gene expression data using real-time quantitative PCR and the 2(-Delta Delta C(T)) method. methods 25: 402-408, 2001.

20. Bustin SA, Benes V, Garson J, Hellemans J, Huggett J, Kubista M, Mueller R, Nolan T, Pfaffl MW, Shipley G, et al: The need for transparency and good practices in the qPCR literature. Nat Methods 10: 1063-1067, 2013.

21. Wishart DS, Knox C, Guo AC, Cheng D, Shrivastava S, Tzur D, Gautam B and Hassanali M: DrugBank: A knowledgebase for drugs, drug actions and drug targets. Nucleic Acids Res 36 (Database issue): D901-D906, 2008.

22. Keshava Prasad TS, Goel R, Kandasamy K, Keerthikumar S, Kumar S, Mathivanan S, Telikicherla D, Raju R, Shafreen B, Venugopal A, et al: Human protein reference database-2009 update. Nucleic Acids Res 37 (Database issue): D767-D772, 2009.

23. Stark C, Breitkreutz BJ, Reguly T, Boucher L, Breitkreutz A and Tyers M: BioGRID: A general repository for interaction datasets. Nucleic Acids Res 34 (Database issue): D535-D539, 2006. 
24. Aranda B, Achuthan P, Alam-Faruque Y, Armean I, Bridge A, Derow C, Feuermann M, Ghanbarian AT, Kerrien S, Khadake J, et al: The IntAct molecular interaction database in 2010. Nucleic Acids Res 38 (Database issue): D525-D531, 2010.

25. Ceol A, Chatr Aryamontri A, Licata L, Peluso D, Briganti L, Perfetto L, Castagnoli L and Cesareni G: MINT, the molecular interaction database: 2009 update. Nucleic Acids Res 38 (Database issue): D532-D539, 2010

26. Salwinski L, Miller CS, Smith AJ, Pettit FK, Bowie JU and Eisenberg D: The Database of interacting proteins: 2004 update. Nucleic Acids Res 32 (Database issue): D449-D451, 2004.

27. Gibson TA and Goldberg DS: Questioning the ubiquity of neofunctionalization. PLoS Comput Biol 5: e1000252, 2009.

28. Kanehisa M and Goto S: KEGG: Kyoto encyclopedia of genes and genomes. Nucleic Acids Res 28: 27-30, 2000.

29. Ashburner M, Ball CA, Blake JA, Botstein D, Butler H, Cherry JM, Davis AP, Dolinski K, Dwight SS, Eppig JT, et al: Gene ontology: Tool for the unification of biology. The Gene Ontology Consortium. Nat Genet 25: 25-29, 2000.

30. Allen DG, Gervasio OL, Yeung EW and Whitehead NP: Calcium and the damage pathways in muscular dystrophy. Can J Physiol Pharmacol 88: 83-91, 2010.

31. Kang C, Goodman CA, Hornberger TA and Ji LL: PGC-10 overexpression by in vivo transfection attenuates mitochondrial deterioration of skeletal muscle caused by immobilization. FASEB J 29: 4092-4106, 2015

32. Peter JB, Verhaag DA and Worsfold M: Studies of steroid myopathy. Examination of the possible effect of triamcinolone on mitochondria and sarcotubular vesicles of rat skeletal muscle. Biochem Pharmacol 19: 1627-1636, 1970.

33. Cannavino J, Brocca L, Sandri M, Grassi B, Bottinelli R and Pellegrino MA: The role of alterations in mitochondrial dynamics and PGC-1 $\alpha$ over-expression in fast muscle atrophy following hindlimb unloading. J Physiol 593: 1981-1995, 2015.

34. Lynch CJ, Xu Y, Hajnal A, Salzberg AC and Kawasawa YI: RNA sequencing reveals a slow to fast muscle fiber type transition after olanzapine infusion in rats. PLoS One 10: e0123966, 2015.

35. Foster RA and Willis MC: Tandem inverse-electron-demand hetero-/retro-Diels-Alder reactions for aromatic nitrogen heterocycle synthesis. Chem Soc Rev 42: 63-76, 2013.

36. Georgiou DK, Dagnino-Acosta A, Lee CS, Griffin DM, Wang H, Lagor WR, Pautler RG, Dirksen RT and Hamilton SL: Ca2+ binding/permeation via calcium channel, $\mathrm{CaV} 1.1$, regulates the intracellular distribution of the fatty acid transport protein, CD36, and fatty acid metabolism. J Biol Chem 290: 23751-23765, 2015.

37. Pomiès P, Rodriguez J, Blaquière $M$, Sedraoui S, Gouzi $F$ Carnac G, Laoudj-Chenivesse D, Mercier J, Préfaut C and Hayot M: Reduced myotube diameter, atrophic signalling and elevated oxidative stress in cultured satellite cells from COPD patients. J Cell Mol Med 19: 175-186, 2015.

38. Nabuurs CI, Choe CU, Veltien A, Kan HE, van Loon LJ Rodenburg RJ, Matschke J, Wieringa B, Kemp GJ, Isbrandt D and Heerschap A: Disturbed energy metabolism and muscular dystrophy caused by pure creatine deficiency are reversible by creatine intake. J Physiol 591: 571-592, 2013.

39. Kramer A, Mulhauser F, Wersig C, Gröning K and Bilbe G: Mammalian splicing factor SF3a120 represents a new member of the SURP family of proteins and is homologous to the essential splicing factor PRP21p of Saccharomyces cerevisiae. RNA 1: 260-272, 1995

40. Perez R, Tupac-Yupanqui I and Dunner S: Evaluation of suitable reference genes for gene expression studies in bovine muscular tissue. BMC Mol Biol 9: 79, 2008.

41. Ramayo-Caldas Y, Fortes MR, Hudson NJ, Porto-Neto LR, Bolormaa S, Barendse W, Kelly M, Moore SS, Goddard ME, Lehnert SA and Reverter A: A marker-derived gene network reveals the regulatory role of PPARGC1A, HNF4G, and FOXP3 in intramuscular fat deposition of beef cattle. J Anim Sci 92: 2832-2845, 2014

42. Sakuma K, Nakao R, Inashima S, Hirata M, Kubo T and Yasuhara M: Marked reduction of focal adhesion kinase, serum response factor and myocyte enhancer factor $2 \mathrm{C}$, but increase in RhoA and myostatin in the hindlimb dy mouse muscles. Acta Neuropathol 108: 241-249, 2004.

43. Davegårdh C, Broholm C, Perfilyev A, Henriksen T, García-Calzón S, Peijs L, Hansen NS, Volkov P, Kjøbsted R, Wojtaszewski JF, et al: Abnormal epigenetic changes during differentiation of human skeletal muscle stem cells from obese subjects. BMC Med 15: 39, 2017.
44. Arosio A, Sala G, Rodriguez-Menendez V, Grana D, Gerardi F, Lunetta C, Ferrarese C and Tremolizzo L: MEF2D and MEF2C pathways disruption in sporadic and familial ALS patients. Mol Cell Neurosci 74: 10-17, 2016.

45. Snijders T, Wall BT, Dirks ML, Senden JM, Hartgens F, Dolmans J, Losen M, Verdijk LB and van Loon LJ: Muscle disuse atrophy is not accompanied by changes in skeletal muscle satellite cell content. Clin Sci (Lond) 126: 557-566, 2014.

46. Ivannikov MV and Macleod GT: Mitochondrial free $\mathrm{Ca}^{2}(+)$ levels and their effects on energy metabolism in Drosophila motor nerve terminals. Biophys J 104: 2353-2361, 2013.

47. Yamamoto H, Williams EG, Mouchiroud L, Cantó C, Fan W, Downes M, Héligon C, Barish GD, Desvergne B, Evans RM, et al: NCoR1 is a conserved physiological modulator of muscle mass and oxidative function. Cell 147: 827-839, 2011.

48. Blaauw B, Schiaffino S and Reggiani C: Mechanisms modulating skeletal muscle phenotype. Compr Physiol 3: 1645-1687, 2013.

49. Nierobisz LS, Cheatham B, Buehrer BM and Sexton JZ: High-content screening of human primary muscle satellite cells for new therapies for muscular atrophy/dystrophy. Curr Chem Genom Transl Med 7: 21-29, 2013

50. Grounds MD: Therapies for sarcopenia and regeneration of old skeletal muscles: More a case of old tissue architecture than old stem cells. Bioarchitecture 4: 81-87, 2014

51. Cannavino J,Brocca L, Sandri M, Bottinelli R and Pellegrino MA: PGC1- $\alpha$ over-expression prevents metabolic alterations and soleus muscle atrophy in hindlimb unloaded mice. J Physiol 592: 4575-4589, 2014

52. Brocca L, Pellegrino MA, Desaphy JF, Pierno S, Camerino DC and Bottinelli R: Is oxidative stress a cause or consequence of disuse muscle atrophy in mice? A proteomic approach in hindlimb-unloaded mice. Exp Physiol 95: 331-350, 2010.

53. Momken I, Stevens L, Bergouignan A, Desplanches D, Rudwill F, Chery I, Zahariev A, Zahn S, Stein TP, Sebedio JL, et al: Resveratrol prevents the wasting disorders of mechanical unloading by acting as a physical exercise mimetic in the rat. FASEB J 25: 3646-3660, 2011.

54. Romanello V, Guadagnin E, Gomes L, Roder I, Sandri C, Petersen Y, Milan G, Masiero E, Del Piccolo P, Foretz M, et al: Mitochondrial fission and remodelling contributes to muscle atrophy. EMBO J 29: 1774-1785, 2010.

55. Romanello V and Sandri M: Mitochondrial biogenesis and fragmentation as regulators of muscle protein degradation. Curr Hypertens Rep 12: 433-439, 2010.

56. Alenghat T, Meyers K, Mullican SE, Leitner K, Adeniji-Adele A, Avila J, Bućan M, Ahima RS, Kaestner KH and Lazar MA: Nuclear receptor corepressor and histone deacetylase 3 govern circadian metabolic physiology. Nature 456: 997-1000, 2008.

57. Potthoff MJ and Olson EN: MEF2: A central regulator of diverse developmental programs. Development 134: 4131-4140, 2007.

58. Gao YF, Fan XL, He ZX, Wu SD and Song XA: Effects of Ligustrazine and Radix Astragali on activities of myosin adenosine triphosphatase of soleus muscle and muscle atrophy in tail-suspended rat. Space Med Med Eng (Beijing) 18: 262-266, 2005.

59. Tabandeh MR, Jafari H, Hosseini SA and Hashemitabar M: Ginsenoside $\mathrm{Rb} 1$ stimulates adiponectin signaling in $\mathrm{C} 2 \mathrm{C} 12$ muscle cells through up-regulation of AdipoR1 and AdipoR2 proteins. Pharm Biol 53: 125-132, 2015.

60. Lustig Y, Barhod E, Ashwal-Fluss R, Gordin R, Shomron N, Baruch-Umansky K, Hemi R, Karasik A and Kanety H: RNA-binding protein PTB and microRNA-221 coregulate AdipoR1 translation and adiponectin signaling. Diabetes 63: 433-445, 2014.

61. Tian JS, Peng GJ, Gao XX, Zhou YZ, Xing J, Qin XM and Du GH: Dynamic analysis of the endogenous metabolites in depressed patients treated with TCM formula Xiaoyaosan using urinary (1)H NMR-based metabolomics. J Ethnopharmacol 158: 1-10, 2014.

62. Wang XL, Wang T and Wang YN: Effects of angelica polysaccharides on the proliferation of mouse skeletal muscle satellite cells and the expression of stem cell factor receptor protein. Zhongguo Zhong Xi Yi Jie He Za Zhi 32: 93-96, 2012 (In Chinese). 